\title{
¿Existe un efecto de borde sobre la estructura vegetal y el potencial de regeneración en fragmentos de bosque seco tropical?
}

Is there an edge effect on the vegetation structure and regeneration potential of tropical dry forest fragments?

\author{
Natalia Henao, Alba M. Torres, Juan C. Tafur y Leonardo Guevara
}

\section{Resumen}

La fragmentación es definida como el proceso de trasformación de un hábitat natural en parches de menor área, aislados entre sí e inmersos en matrices de menor cobertura vegetal. Los parches resultantes quedan expuestos a las condiciones del ambiente exterior o efectos de borde, que pueden ocasionar cambios en la estructura y regeneración de las comunidades vegetales. En esta investigación se evaluó el efecto de borde sobre la estructura, diversidad y potencial de regeneración de plantas leñosas en dos fragmentos de bosque seco. Se registró un proceso activo de regeneración natural, sin embargo, no se encontró un efecto de borde sobre las variables estudiadas para ambos fragmentos. La ausencia de efecto de borde podría estar dada por el tiempo transcurrido desde la formación del fragmento. Durante este tiempo han estado activos los procesos de regeneración, dando lugar a una vegetación de estructura y composición similar en todo el bosque. También se observó que las matrices de estos fragmentos constituyen una barrera para su expansión y conectividad, y para superarla se hace necesaria la implementación de estrategias que favorezcan la dispersión de semillas hacia el exterior y, se optimicen las condiciones para la germinación y el establecimiento de los nuevos propágulos.

Palabras clave. Efecto de borde. Fragmentación. Juveniles. Plántulas. Reclutamiento.

\begin{abstract}
Fragmentation is defined as the transformation of natural habitats into smaller forest patches that are isolated and immersed in matrices of reduced vegetation cover. The derived patches are exposed to the conditions of the external environment or edge effects, which can cause changes on the structure of plant communities and their regeneration. In this study, we evaluated the effect of the distance to the edge on the structure, diversity and regeneration potential of woody plants in two dry forest fragments. An active process of natural regeneration was registered; nevertheless, an edge effect on the studied variables for both fragments was not evidenced. The absence of an edge effect might be given by the time passed from the formation of the fragment, during which regeneration processes have produced vegetation that is similar in composition and structure to the whole forest. It was also observed that the matrices that surround these fragments constitute a barrier for their expansion and connectivity. To overcome such barrier, it is necessary to implement strategies that favor the dispersion of seeds towards the exterior and optimize the conditions for the germination and the establishment of the new individuals.
\end{abstract}

Keywords. Edge effect. Fragmentation. Recruitment. Sapling. Seedlings. 


\section{Introducción}

Por sus características climáticas y edafológicas, los bosques secos tropicales (BST) han sido una zona de vida preferida para la expansión urbana y agrícola (Murphy y Lugo, 1986). Esto los ha llevado a un avanzado estado de fragmentación en distintas regiones del mundo (Miles et al., 2006), y los ha situado como uno de los ecosistemas más amenazados a nivel mundial (Janzen, 1988; Pizano y García, 2014). En Colombia, solo permanece el 8 $\%$ del ecosistema original, en forma de fragmentos pobremente representados en el Sistema Nacional de Áreas Protegidas (García et al., 2014). En el caso de la cuenca alta del río Cauca, 14 de 32 fragmentos reportados (Lozano et al., 2007), están en buen estado de conservación, y solo unos cuantos están bajo una figura de protección pública o privada (Arcila et al., 2012; García et al., 2014).

La fragmentación es definida como el proceso de trasformación de un hábitat natural en un número de parches boscosos de menor área, aislados entre sí y en su mayoría inmersos en matrices productivas de menor cobertura vegetal (p. ej. agrícola, ganadera, urbana) (Fahrig, 2003). En consecuencia, las plantas y otros organismos que habitan las zonas externas de estos fragmentos (i.e bordes) quedan expuestos a los llamados efectos de borde, que representan los efectos causados por las condiciones bióticas y abióticas de los ambientes circundantes (i.e. matrices) sobre las condiciones del fragmento (Murcia, 1995; Ries et al., 2004). Los primeros efectos de borde ocurren a nivel estructural del bosque, como resultado del daño y derribamiento de árboles, alteración del suelo, y cambios en el ciclaje de nutrientes (Saunders et al., 1991). De esta manera, en la zona del bosque que limita con la matriz se presenta un microclima de menor humedad y mayor temperatura del aire y del suelo, en comparación con el interior del bosque, donde hay mayor humedad y menor temperatura (Chen et al., 1999). Debido a la alteración de los gradientes bióticos y abióticos originales, también se generan respuestas secundarias sobre los distintos patrones y procesos ecológicos de las plantas, como la regeneración, crecimiento, reproducción y mortalidad (Harper et al., 2005). De estos factores, las dinámicas de regeneración natural juegan un papel muy importante en la capacidad que tienen las comunidades vegetales de renovarse en el tiempo y el espacio.

La regeneración natural abarca desdela producción de semillas viables y su dispersión, hasta su germinación, establecimiento y sobrevivencia (i.e. plántulas, juveniles) (Grubb, 1977; Norden, 2014), pero es durante el establecimiento y sobrevivencia de las plántulas cuando se presentan los filtros más fuertes para la regeneración de un bosque (Poorter y Markesteijn, 2008; Sampaio y Scariot, 2011). Dentro de estos se encuentran los filtros dados por las condiciones del medio ambiente (disponibilidad de agua, luz, o nutrientes en el suelo), filtros a nivel fisiológico y funcional de las plántulas (Cavender-Bares y Bazzaz, 2000; Williams-Linera y Lorea, 2009), y filtros ecológicos resultantes de los procesos dependientes de la densidad y proximidad entre individuos de su misma especie y de otras especies (Comita et al., 2014; Dewalt et al., 2015). Por consiguiente, la alteración de los gradientes bióticos y abióticos que ocurren con la fragmentación también puede afectar la posibilidad que tiene una comunidad vegetal de reproducirse y permanecer en el tiempo (i.e. potencial de regeneración).

Dada la importancia que tiene el potencial de regeneración para la reproducción y permanencia de un bosque, algunas investigaciones se han enfocado en estudiarlo en condiciones de perturbación natural y antrópica. Se han evaluado la densidad, composición y estrategias de las especies del banco de semillas, con el fin de conocer el potencial de regeneración de áreas afectadas por la presencia de una especie invasora (Brandão et al., 2016). Otras investigaciones se han enfocado en evaluar el potencial de regeneración y la distribución de especies, ya sea en fragmentos que difieren en el tamaño y el tipo de matriz (Botzat et al., 2015), o bajo las diferentes condiciones climáticas y edáficas previstas con el 
cambio climático (Mok et al., 2012). Sin embargo, aún no se ha evaluado el cambio de este potencial respecto a los efectos de borde, y en qué medida la composición de plántulas y juveniles representa la composición de la etapa adulta.

Como se mencionó anteriormente, la sobrevivencia de las plántulas puede verse afectada por los cambios ambientales e interacciones bióticas, incluidos aquellos ocasionados por la fragmentación. Varios autores han reportado diferencias en la riqueza y densidad de individuos de la etapa regenerativa entre borde e interior de bosque (Sizer y Tanner, 1999; Sampaio y Scariot, 2011). También se ha encontrado que, en los bordes de los fragmentos, la regeneración puede ser menos exitosa debido a que hay una mayor tasa de depredación de semillas, herbivoría sobre plántulas y ataque de patógenos (Benítez-Malvido y Lemus-Albor, 2005; Vieira y Scariot, 2006). Por otro lado, se ha encontrado que las condiciones ambientales de un sitio y las adaptaciones de las especies a estos ambientes (Sampaio y Scariot, 2011), así como las altas tasas de regeneración natural (Bouroncle y Finegan, 2011), pueden incidir en la disminución del efecto de borde sobre las comunidades vegetales. En este sentido, el efecto de borde sobre la diversidad y regeneración de fragmentos de bosque constituye un tema importante para aportar información sobre la capacidad de regeneración de estos fragmentos y cuál es su valor para la continuidad de los ecosistemas que representan.

Teniendo en cuenta el deterioro del BST, el conocimiento de la dinámica de regeneración en estos fragmentos también permitirá orientar algunas de las estrategias propuestas para la restauración de los BST, tales como el enriquecimiento de especies y la ampliación de las fronteras de estos fragmentos (Murphy y Lugo, 1986; Vargas y Ramírez, 2014). Dado lo anterior, esta investigación se enfocó en evaluar el efecto de la distancia al borde sobre la estructura vegetal, diversidad y potencial de regeneración de dos fragmentos de BST en el Valle del Cauca, Colombia. En primer lugar, se evaluó si la estructura y diversidad de las plántulas y juveniles (i.e. etapa de regeneración) y los adultos de especies leñosas, cambiaban conforme se avanzaba desde el borde hasta el interior de los fragmentos. También se propuso una medida del potencial de regeneración en términos de la representatividad de las etapas de plántula y juvenil con relación a la etapa adulta, y se evaluó si este potencial cambiaba en un gradiente de distancia desde el borde hasta el interior de los fragmentos. Finalmente, se indagó si estos cambios se manifestaban a nivel de la composición de especies en las etapas de regeneración y adulto.

\section{Materiales y métodos}

Área de estudio. Este estudio se realizó entre noviembre de 2014 y enero de 2015, en dos fragmentos de BST que se encuentran en el Parque Natural Regional (PNR) El Vínculo $\left(3^{\circ} 50^{\prime} 23^{\prime \prime} \mathrm{N}-76^{\circ} 18^{\prime} 07^{\prime \prime} \mathrm{O}\right)$ y en la Reserva Natural de la Sociedad Civil (RNSC) El Hatico (033' $\left.39^{\prime \prime} \mathrm{N}-76^{\circ} 19^{\prime} 28,8^{\prime \prime} \mathrm{O}\right)$, y localizados en el departamento del Valle del Cauca, Colombia (Figuras 1 y 2).

El PNR El Vínculo está ubicado en el piedemonte de la cordillera Central en el corregimiento El Vínculo, al sur del municipio de Buga, Valle del Cauca. Su altitud varía entre 977 y 1150 m s.n.m., presenta una temperatura promedio de $24{ }^{\circ} \mathrm{C}$ y $1379 \mathrm{~mm}$ de precipitación promedio anual. El clima es bimodal con dos periodos lluviosos (marzo-mayo y septiembre-noviembre) y dos secos (enero-febrero y julio-agosto). El parque tiene un área aproximada de 74 ha, de las cuales 34 ha corresponden a un fragmento de bosque que se ha preservado de la explotación agropecuaria desde 1969, favoreciendo la regeneración natural (Torres et al., 2012). La parte alta del bosque presenta vegetación arbustiva, mientras que la parte baja presenta árboles maduros y de mayor porte. Este bosque se encuentra rodeado principalmente por una matriz de pastizales arbolados y caña de azúcar. El bosque se encuentra en un estado sucesional temprano y algunas de las especies dominantes son Eugenia procera, Amyris pinnata, 


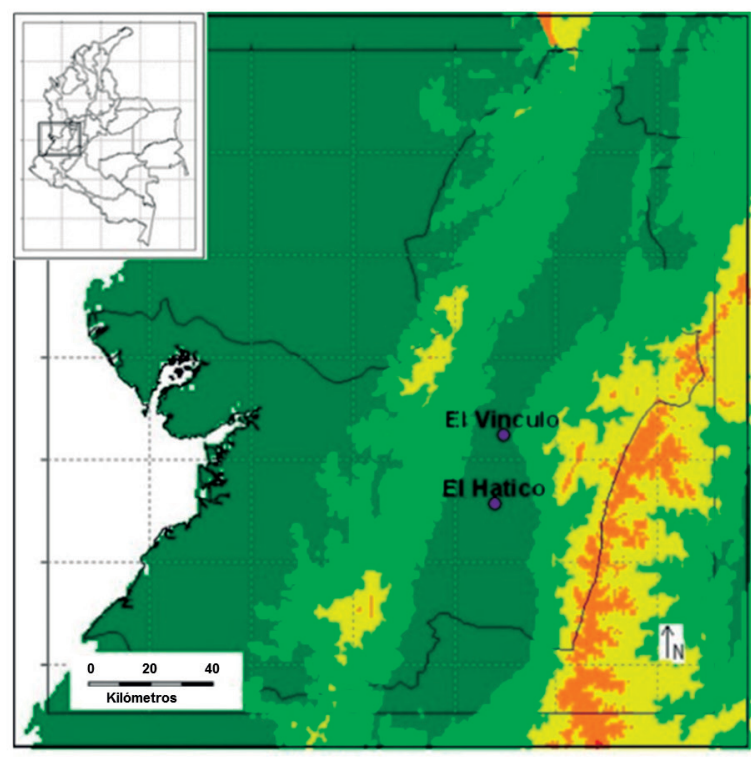

(a)

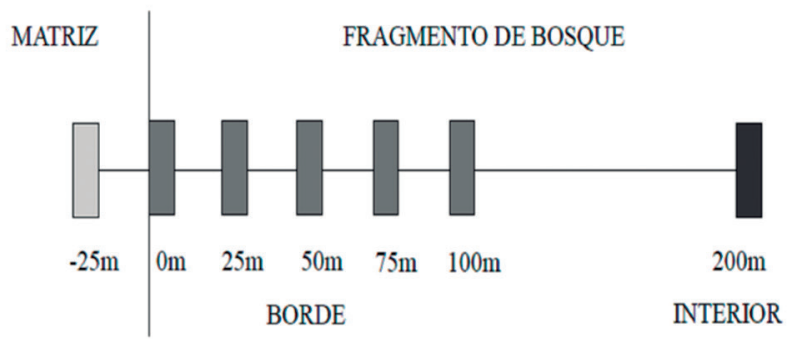

(b)

Figura 1. A) Ubicación de los fragmentos de BST del PNR El Vínculo y la RNSC El Hatico en el Valle del Cauca, Colombia. B) Esquema del diseño del muestreo.

Pithecellobium lanceolatum, Guapira sp. y Guazuma ulmifolia (Torres et al., 2012).

La RNSC El Hatico está localizada en el municipio de El Cerrito, Valle del Cauca, tiene una extensión de 288 ha donde hay un fragmento de bosque de aproximadamente 14 ha, que ha sido preservado por sus dueños desde 1947. Su altitud varía entre 900 y 1000 m s.n.m., presenta una temperatura promedio de $24{ }^{\circ} \mathrm{C}$ y $750 \mathrm{~mm}$ de precipitación promedio anual (Molina et al., 2012). El bosque está rodeado por guaduales nativos y cultivados, zonas de sucesión vegetal, un corredor biológico, sistemas silvopastoriles y cultivos de caña de azúcar orgánica (Molina et al., 2012). Su dosel es relativamente alto y continuo, con especies como Anacardium excelsum, Ficus insipida, Ceiba pentandra y Samanea saman.

Caracterización vegetal. En cada fragmento se establecieron cuatro transectos de $225 \mathrm{~m}$, separados $200 \mathrm{~m}$ entre sí y perpendiculares al borde. Se asumió como borde el límite donde cambia la composición del bosque y empieza la matriz, sin tener en cuenta el alcance del dosel
(Murcia, 1995). En cada transecto se demarcaron 6 parcelas de $10 \times 2 \mathrm{~m}$ a intervalos de $25 \mathrm{~m}$, iniciando desde la matriz $(-25 \mathrm{~m})$ hasta los $100 \mathrm{~m}$ del borde y 1 parcela de igual tamaño a $200 \mathrm{~m}$ en el interior del fragmento (Laurance et al., 2002; Lopes et al., 2009) (Figura 3).

En cada parcela se registraron los individuos con un diámetro a la altura del pecho $(\mathrm{DAP}) \geq 2,5 \mathrm{~cm}$ (medido a 1,3 m del suelo) y se clasificaron como adultos. Para cada individuo se registró el DAP $(\mathrm{cm})$ y la altura total $(\mathrm{m})$. También se contaron todas las plántulas (circunferencia $<1 \mathrm{~cm}$ y altura $<110 \mathrm{~cm}$ ), y juveniles (circunferencia entre 1 y 2,5 $\mathrm{cm})$, y se marcaron con un aro plástico y una cinta llamativa rotulada con el número del individuo. Las plántulas y juveniles fueron identificadas hasta el menor nivel taxonómico posible con ayuda de claves taxonómicas, revisión de material de herbario y comparación con individuos adultos que se encontraron cerca del lugar de muestreo. Cuando fue necesario, se colectaron especímenes para su identificación y las muestras fueron depositadas en el herbario de la Universidad del Valle (CUVC). 

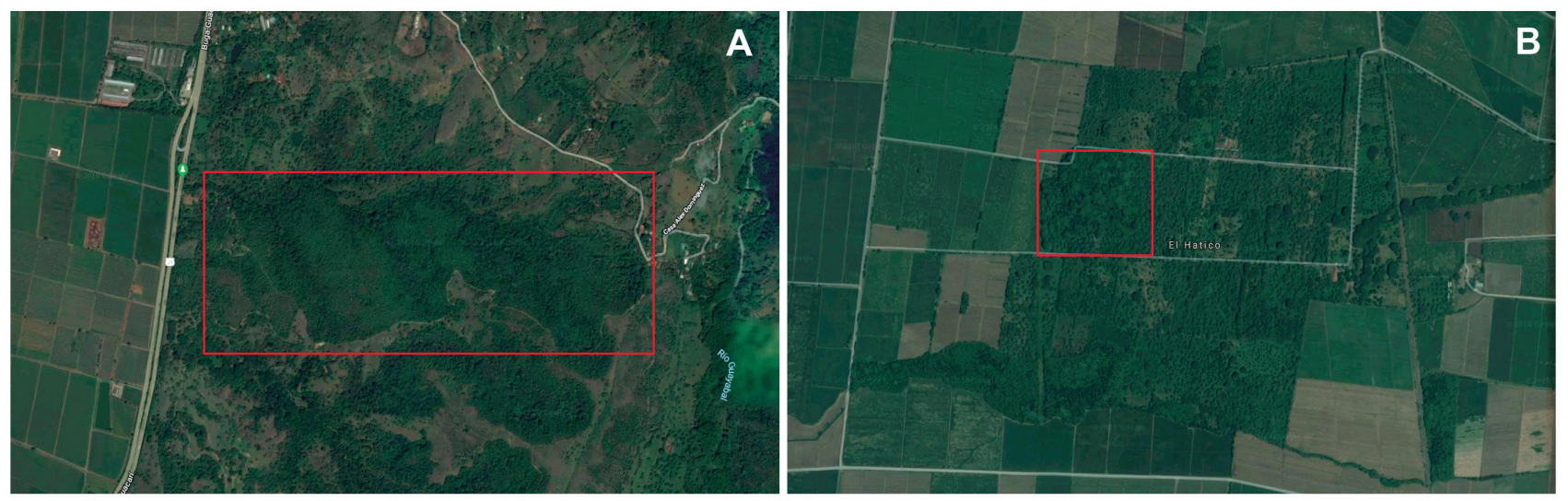

Figura 2. Imágenes satelitales que muestran el contexto espacial donde están ubicados los fragmentos de BST del PNR El Vínculo A) y la RNSC El Hatico B).

EL VÍNCULO
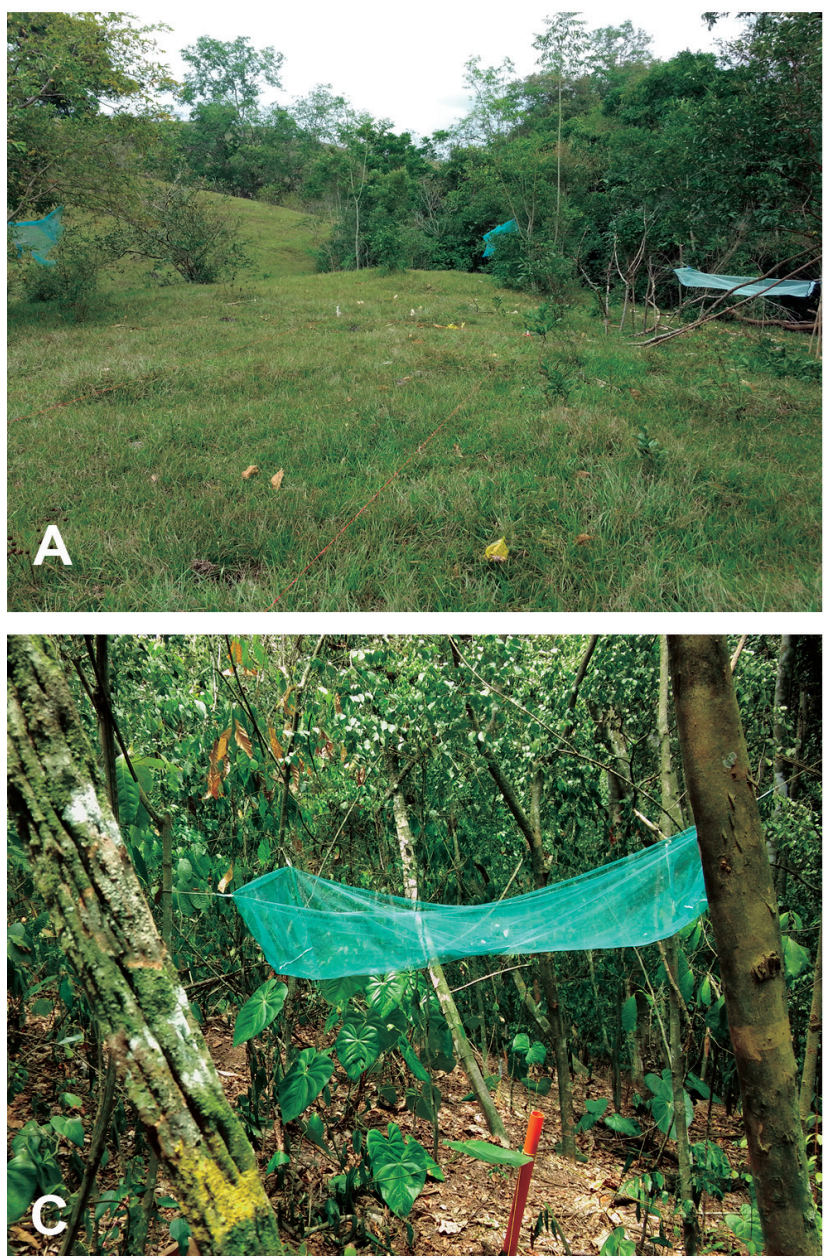

\section{EL HATICO}
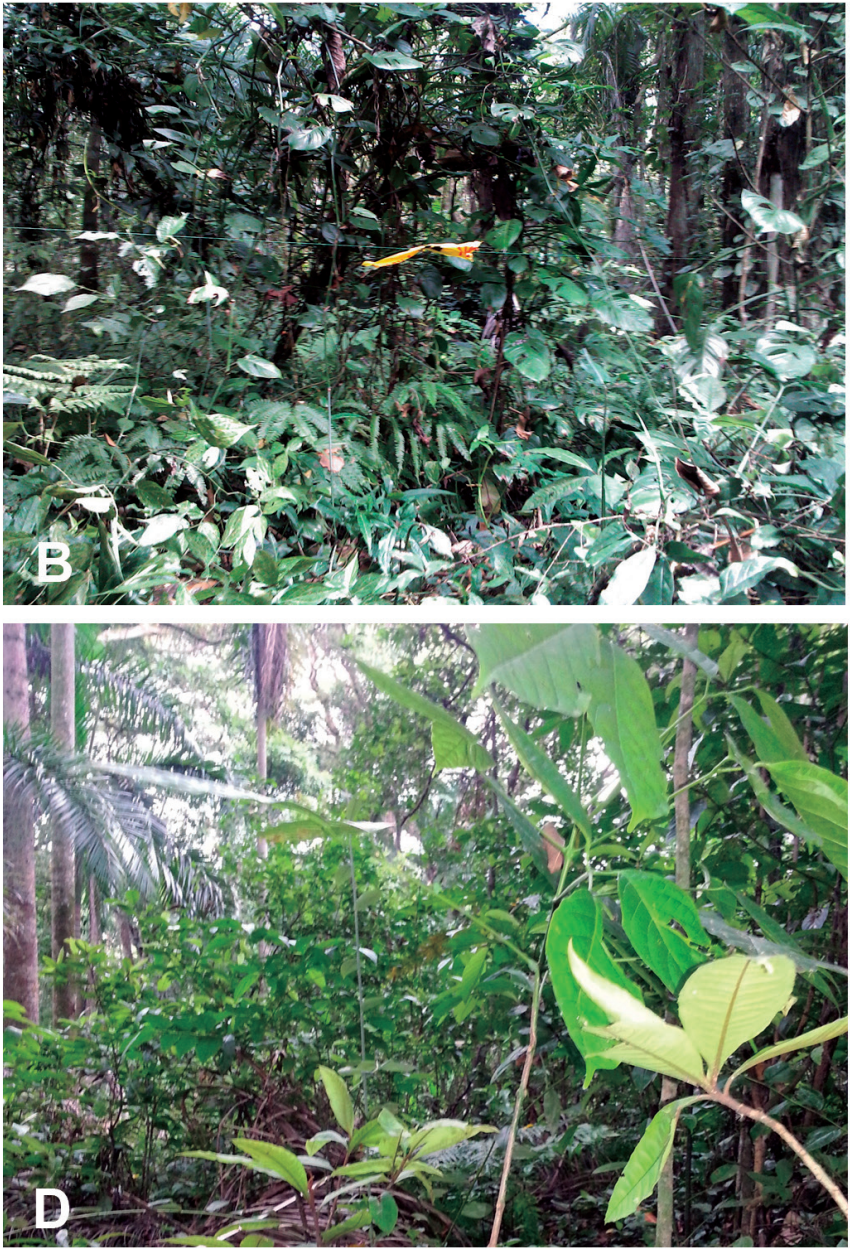

Figura 3. A) Aspecto del borde y C) aspecto del interior del fragmento de BST del PNR El Vínculo. B) Aspecto del borde y D) aspecto del interior de la RNSC El Hatico. 
Análisis de datos de estructura y diversidad. La estructura de la vegetación se evaluó por medio de la densidad de individuos (número de individuos/área de la parcela) para las tres etapas de crecimiento; y para la etapa adulto se evaluó además la altura y el DAP. Se estimó la diversidad de adultos, juveniles y plántulas por parcela, a través de la riqueza (número de especies) y elíndice de diversidad de Shannon-Wiener (Hammer et al., 2001). Para estimar el efecto de la distancia desde el borde (variable explicativa) se evaluó la diferencia en la estructura y diversidad entre parcelas con Modelos Lineales Generalizados (GLM). Este tipo de modelos permite realizar análisis de varianza (ANOVA) cuando los datos de la variable de respuesta son conteos o no siguen los supuestos de la normalidad (Faraway, 2016). En estos modelos también se incluyeron los resultados de las parcelas correspondientes a la matriz (-25 $\mathrm{m})$, con el fin de compararlas con las parcelas de bosque. Las variables de conteo se ajustaron a una distribución de Poisson con la función vínculo Log. En los casos en que se presentó una significancia estadística $(p<0,05)$ se realizó una prueba de Tukey para detectar las distancias al borde en que se presentaron los cambios. Los análisis fueron realizados con el programa estadístico $\mathrm{R}$ ( $\mathrm{R}$ Core Team, 2014).

Análisis de datos de potencial de regeneración. Para estimar el potencial de regeneración $\left(\mathrm{P}_{\mathrm{Reg}}\right)$ en los fragmentos, se determinó, para cada parcela el número de especies de las etapas de regeneración (i.e. plántulas, juveniles) compartidas con el total de las especies de adultos registrados en todas las parcelas, con el objetivo de representar la mayor diversidad de adultos posibles. De esta manera, el rango del $\mathrm{P}_{\text {Reg }}$ oscilaba entre 0 y $100 \%$. El valor máximo alcanzado indicaba que todas las especies adultas estaban representadas en las etapas de regeneración. Se usó la siguiente fórmula: $P_{\text {Reg }}=\frac{\text { (n. de especies de plántulas }+n \text {. de especies de juveniles }) \times 100}{n \text {. de especies de adultos }}$
Con el fin de evaluar si hubo un efecto de la distancia desde el borde sobre el potencial de regeneración, se realizaron Modelos Lineales Generalizados (GLM). La variable $P_{\text {Reg }}$ se ajustó para una distribución binomial. En los casos en que se presentó una significancia estadística $(p<$ $0,05)$ se realizó una prueba de Tukey para detectar las distancias al borde en que se presentaron los cambios.

Mediante un escalamiento no métrico multidimensional (NMDS) se comparó la composición de la etapa de regeneración y la etapa adulta, con el fin de evaluar cambios a lo largo del gradiente de borde a interior. Las diferencias entre los grupos se evaluaron mediante una PERMANOVA (Hammer et al., 2001).

\section{Resultados}

Se registraron en total 68 especies de plantas, de las cuales 42 estuvieron presentes en El Vínculo y 38 en El Hatico. Se contaron 1125 individuos para El Vínculo y 2165 para El Hatico, de todas las etapas evaluadas. Para los 2 fragmentos, la abundancia de individuos en la etapa adulta fue similar (Tabla 1). En cambio, la abundancia de juveniles fue mayor en El Vínculo que en El Hatico. Aunque la etapa plántula fue la más abundante en los dos fragmentos, su abundancia fue mayor en El Hatico. Los dos fragmentos presentaron un número de familias similar (21 en El Vínculo y 22 en El Hatico) (Anexo 1). En general, para los 2 fragmentos las familias con el mayor número de especies fueron Rubiaceae (11 especies) y Fabaceae (8). En El Vínculo, las familias Rubiaceae y Fabaceae registraron 5 especies cada una, seguidas por Rutaceae (4) y Sapindaceae (4). En El Hatico sobresale la familia Rubiaceae (8), seguida por Fabaceae (4) y Meliaceae (3).

En El Vínculo, las especies más abundantes en la etapa adulta fueron Eugenia procera $(38,2 \%)$, E. monticola $(20,7 \%)$ y Sorocea sprucei $(9,7 \%)$; las especies restantes se registraron en una cantidad inferior al $5 \%$. En la etapa juvenil se destacan Brosimum alicastrum (21,2 \%), Ocotea veraguensis 
Tabla 1. Riqueza y abundancia de plántulas, juveniles y adultos, en los BST del PNR El Vínculo y la RNSC El Hatico, Valle del Cauca, Colombia.

\begin{tabular}{lcccc}
\hline & \multicolumn{4}{c}{ Fragmentos } \\
\cline { 2 - 5 } & \multicolumn{2}{c}{ El Vínculo } & \multicolumn{2}{c}{ El Hatico } \\
\hline Riqueza & Abundancia & Riqueza & Abundancia \\
\hline Juveniles & 26 & 135 & 12 & 75 \\
\hline Plántulas & 45 & 782 & 24 & 1920 \\
\hline
\end{tabular}

$(18,2 \%)$ y E. procera $(9,6 \%)$. En la etapa plántula, las especies más abundantes fueron $B$. alicastrum $(38,7 \%)$ y O. veraguensis $(16,1 \%)$ (Anexo 1$)$.

En El Hatico Ardisia guianensis (31,7 \%), G. angustifolia (20,6\%) y Anacardium excelsum $(7,6$ $\%)$ fueron las especies más frecuentes en la etapa adulta. En la etapa juvenil A. guianensis fue la especie dominante $(52,9 \%)$ seguida por Psychotria sp. $(27,9 \%)$ y Erythroxylum ulei (7,4 \%). Para las plántulas, la especie más abundante fue Bunchosia pseudonitida (80,6\%), seguida por A. guianensis $(8,1$ $\%)$, A. excelsum $(7,2 \%)$ y E. ulei (6,0 \%) (Anexo 1).

Estructura de los fragmentos. En El Vínculo, la altura de los árboles no presentó diferencias significativas desde el borde $(0 \mathrm{~m})$ hasta el interior (200 m) (Tabla 2). Sin embargo, al incluir la matriz en el análisis si hubo diferencias entre esta y las parcelas de borde a interior (Tukey: $p<0,05$ ). La altura promedio estuvo entre 5-8 m representada en su mayoría por S. sprucei, E. monticola y $E$. procera (Figura 4), y en una menor proporción por árboles de $12 \mathrm{~m}$ de individuos de Genipa americana, G. ulmifolia y Machaerium capote.

Por el contrario, en El Hatico la distribución de alturas de los árboles fue diferente desde el borde hasta el interior (Tabla 2), con diferencias significativas en las parcelas $0 \mathrm{~m}, 75 \mathrm{~m}$ y $100 \mathrm{~m}$ (Tukey: $\mathrm{p}<0,01$ ), que tuvieron mayores alturas por la presencia de individuos de G. angustifolia (16$21 \mathrm{~m})$ y A. excelsum (12 m) (Figura 4). La altura promedio estuvo entre 4 y $10 \mathrm{~m}$, representada principalmente por individuos de A. guianensis, $A$. excelsum y S. sprucei.

El rango promedio de DAP fue $6-10 \mathrm{~cm}$ en ambos fragmentos (Figura 4). En El Vínculo no se presentaron diferencias en el DAP desde el borde $(0 \mathrm{~m})$ hasta el interior $(200 \mathrm{~m})$ (Tabla 2). Sin embargo, en El Hatico se encontraron diferencias significativas en el DAP de la parcela de borde (0 m, Tukey: $p<0,05)$, donde se presentaron los valores más altos de DAP correspondientes a individuos de G. ulmifolia y G. angustifolia.

No se encontraron diferencias significativas en la densidad de adultos desde el borde hasta el interior en los dos fragmentos. En el caso de El Vínculo, solo se presentaron diferencias cuando se incluyó la matriz en el análisis (Tabla 2). En la etapa de regeneración (plántulas y juveniles) la densidad tampoco fue diferente en las distintas distancias para ambos fragmentos (Tabla 2). No obstante, gráficamente se observa que, en El Vínculo, a los 25 $\mathrm{m}$ la abundancia de plántulas es mayor respecto a las otras distancias, mientras que para juveniles se observó una reducción de la densidad a partir de los $75 \mathrm{~m}$ con leve recuperación a los $200 \mathrm{~m}$ (Figura 5). En El Hatico, en la parcela de interior (200 m) se observó una reducción en la densidad de plántulas respecto a las otras distancias, mientras que en la etapa juvenil la densidad se redujo en los $75 \mathrm{~m}$ (Figura 5). 
Tabla 2. Efecto de la distancia desde el borde sobre los parámetros de estructura y diversidad de plántulas, juveniles y adultos en los BST del PNR El Vínculo y la RNSC El Hatico, Valle del Cauca, Colombia. Los números corresponden al valor $p$ de la variable explicativa (distancia desde el borde). Para la evaluación de los modelos se incluyó la matriz (NE: no evaluado).

\begin{tabular}{lccccccc}
\hline & \multicolumn{3}{c}{ El Vínculo } & \multicolumn{3}{c}{ El Hatico } \\
\hline \multicolumn{1}{c}{ Estructura } & Plántulas & Juveniles & Adultos & Plántulas & Juveniles & Adultos \\
\hline Altura & NE & NE & $0,0128^{*}$ & NE & NE & $<0,001^{*}$ \\
\hline DAP & NE & NE & 0,9121 & NE & NE & $<0,001^{*}$ \\
\hline Densidad & 0,5855 & 0,4974 & $<0,001^{*}$ & 0,4437 & 0,2311 & 0,2311 \\
\hline \multicolumn{1}{c}{ Diversidad } & Plántulas & Juveniles & Adultos & Plántulas & Juveniles & Adultos \\
\hline Shannon- Wiener & $0,00336^{*}$ & 0,3222 & $0,0135^{*}$ & $0,0068^{*}$ & 0,3238 & $0,0114^{*}$ \\
\hline Riqueza & $<0,001^{*}$ & $0,0359^{*}$ & 0,2401 & $<0,001^{*}$ & $<0,001^{*}$ & 0,0609 \\
\hline
\end{tabular}

*diferencia significativa $\mathrm{p}<0,05$.

Vínculo
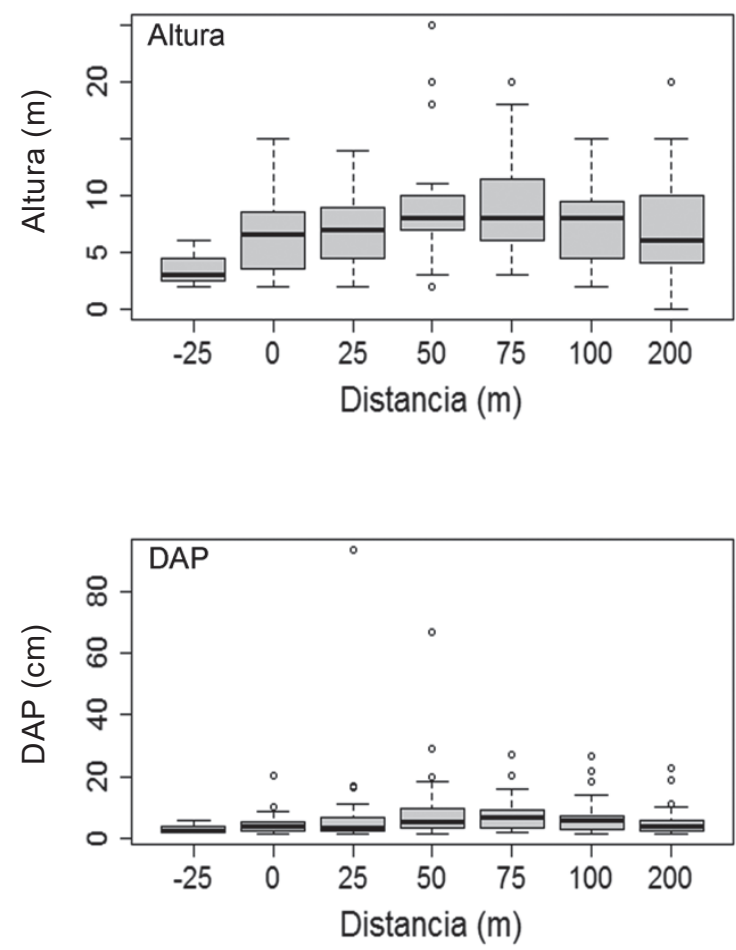

Hatico
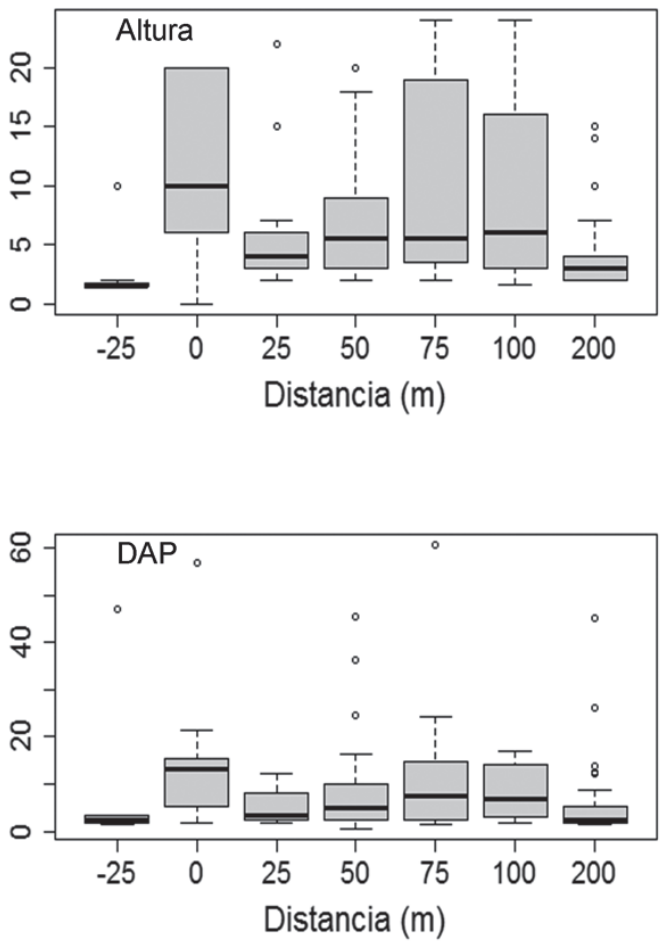

Figura 4. Distribución de la altura y DAP de la clase adulta desde la matriz (-25 m) hacia el interior (200 m) en los fragmentos de BST en el PNR El Vínculo y la RNSC El Hatico, Valle del Cauca, Colombia. 

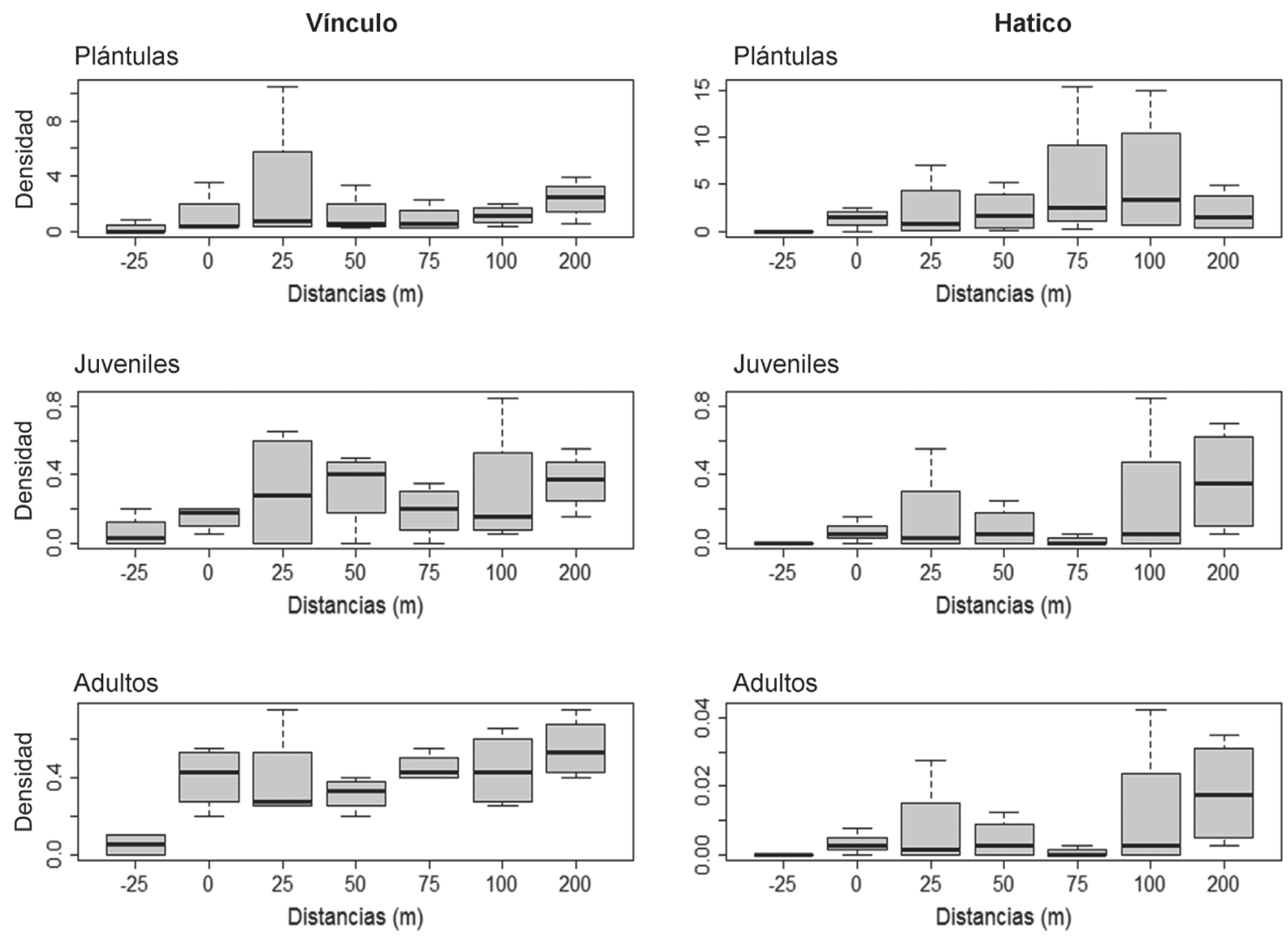

Figura 5. Distribución de la densidad de individuos (\# individuos. $\left.\mathrm{m}^{-2}\right)$ desde la matriz $(-25 \mathrm{~m})$ hacia el interior ( $200 \mathrm{~m}$ ) para las etapas plántula, juvenil y adultos en los fragmentos de BST en el PNR El Vínculo y la RNSC El Hatico, Valle del Cauca, Colombia.

Diversidad en los fragmentos. En los 2 fragmentos, la riqueza de plantas adultas no tuvo diferencias significativas desde el borde hasta el interior (200 m) (Tabla 2). En El Vínculo la riqueza de especies en la etapa juvenil no fue diferente desde el borde al interior y solo fue significativamente menor al comparar con la matriz (Tukey: $p<0,05$ ). En El Hatico, la riqueza de juveniles no tuvo cambios desde la matriz hasta los $75 \mathrm{~m}$ (Figura 5), pero incrementó de manera significativa en las distancias 100 y 200 m (Tukey: $p<0,05$ en ambas parcelas). Para la etapa plántula, en El Vínculo, aunque la riqueza de especies solo fue significativamente menor en la matriz (Tukey: $p<0,05)$, se observó un incremento desde la matriz hasta el interior (Figura 5). Para el caso de El Hatico, la riqueza de especies de plántulas solo fue significativamente menor en la matriz (Tukey: $p<0,05)$, y no presentó cambios desde el borde $(0$ m) hasta el interior (200 m).
En El Vínculo, la diversidad de los individuos adultos no se diferenció significativamente desde el borde hacia el interior (Tabla 2). Sin embargo, al comparar con la matriz (-25 m), la diversidad en esta fue menor respecto a la parcela del interior (Tukey: $p=0,0116$ ). En la etapa plántula, aunque se observa un incremento en la diversidad desde la matriz hacia el interior (200 m), esta solo fue significativa respecto a la matriz en las distancias de 100 y 200 m (Tukey: $p=0,01043$ y $p=0,00208$, respectivamente). No se presentaron diferencias en la diversidad desde el borde al interior para la etapa juvenil (Figura 6). En El Hatico, la diversidad de los individuos adultos presentaron diferencias significativas entre la matriz y las parcelas de $25 \mathrm{~m}$ (Tukey: $p=0,00562$ ) y de $200 \mathrm{~m}$ (Tukey: $p=0,02544$ ). En las plántulas, hubo diferencias significativas entre la diversidad de la parcela de interior (200 m, Tukey: $p<0,001)$, respecto a la matriz. 

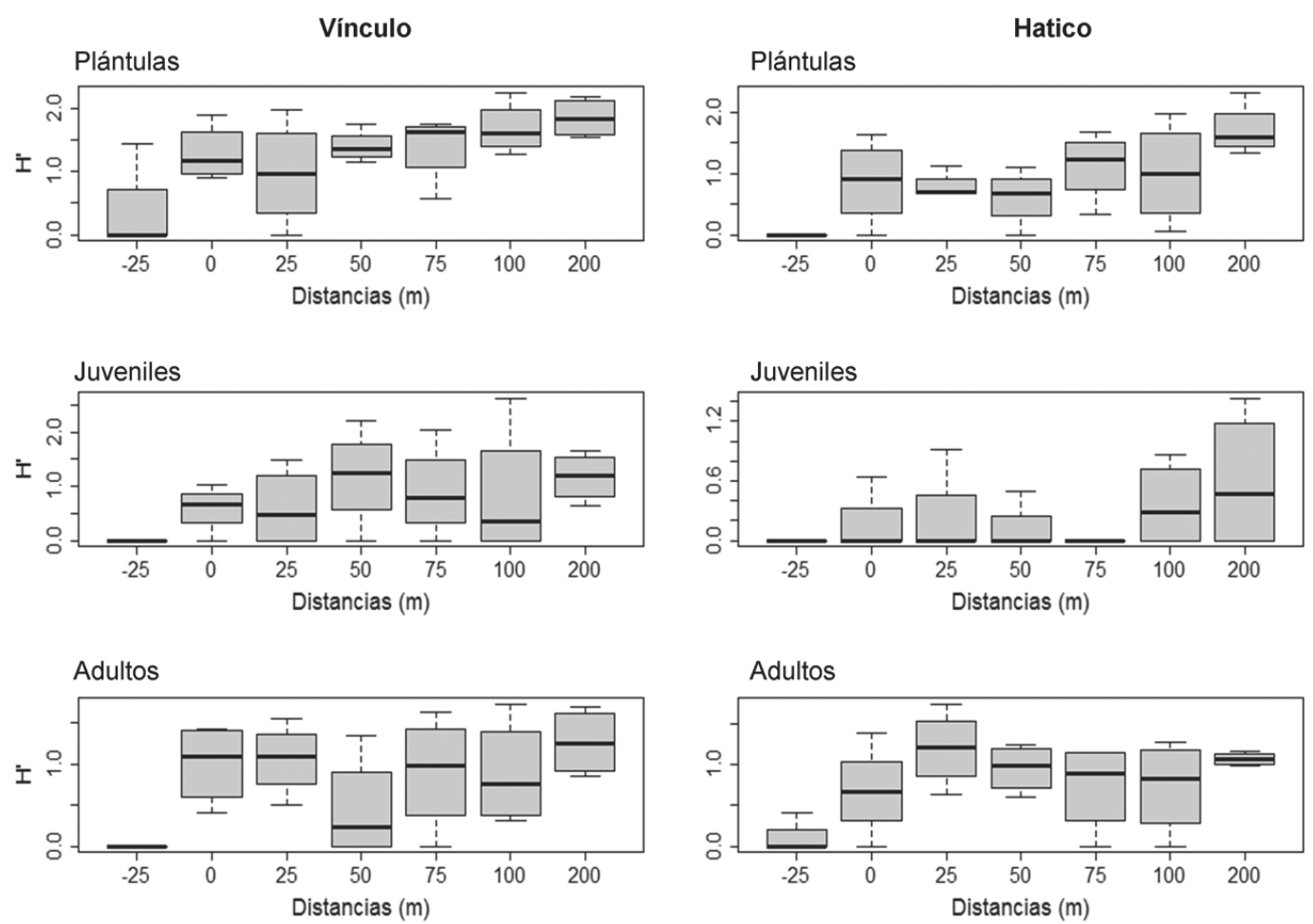

Figura 6. Variación de la diversidad $\mathrm{H}^{\prime}$ (índice de Shannon- Wiener) desde la matriz (-25 $\mathrm{m}$ ) hacia el interior $(200 \mathrm{~m})$ en las etapas plántula, juvenil y adulta en los fragmentos de BST en el PNR El Vínculo y la RNSC El Hatico, Valle del Cauca, Colombia.

Potencial de regeneración en los fragmentos. En los dos fragmentos el $\mathrm{P}_{\text {Reg }}$ fue variable $\mathrm{y}$ presentó diferencias significativas en algunas distancias (Figura 7). En El Vínculo, las parcelas ubicadas a $200 \mathrm{~m}$ tuvieron el mayor valor de $P_{\text {Reg }}$ y se diferenciaron significativamente de las parcelas a $25 \mathrm{~m}$ (Tukey: $p=0,0488$ ). Al tener en cuenta la matriz, el $\mathrm{P}_{\mathrm{Reg}}$ fue más alto en las parcelas de bosque (Tukey: $p<0,05)$. En El Hatico, aunque el $\mathrm{P}_{\mathrm{Reg}}$ fue variable desde el borde $(0 \mathrm{~m})$ hasta el interior $(200 \mathrm{~m})$, las diferencias no fueron significativas y en contraste, la matriz se diferenció significativamente de las parcelas de bosque, siendo casi nula la regeneración en la matriz (Tukey: $p<0,05)$. En términos generales, el $\mathrm{P}_{\mathrm{Reg}}$ varió entre 10 y $31 \%$ en El Vínculo, y entre 8 y 15 \% en El Hatico.
En El Vínculo, la composición de la etapa adulta fue diferente de la composición de las etapas plántula y juvenil (regeneración) $(F=1,589 ; p=$ 0,0013 ). Gráficamente se observa la separación de la etapa adulta y la etapa de regeneración (Figura 8). Sin embargo, en esta gráfica no se observa una separación de acuerdo con las distancias respecto al borde, presentándose parcelas de borde $(0 \mathrm{~m}$, $25 \mathrm{~m})$ cercanas a las parcelas de interior $(100 \mathrm{~m}$, 200 m). En El Hatico, la composición de adultos también fue diferente de la composición de la etapa de regeneración $(F=1,773 ; p=0,001)$. En la gráfica, los puntos de la etapa adulta se ubican hacia la izquierda y los de regeneración a la derecha, sin embargo, no forman grupos definidos de acuerdo con la etapa de vida y tampoco se observa una agrupación de acuerdo con la distancia desde el borde (Figura 8). 

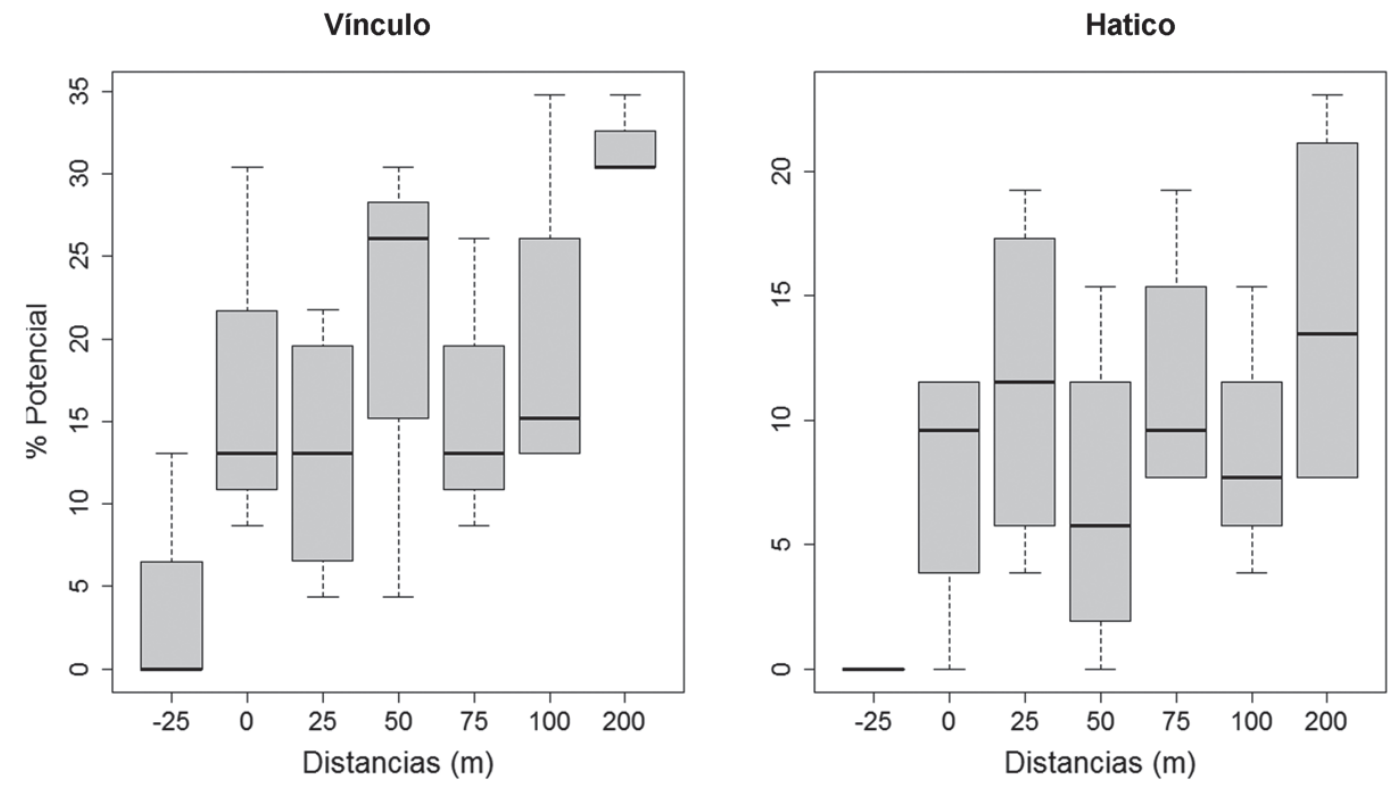

Figura 7. Variación del potencial de regeneración (\%) desde la matriz (-25 m) hacia el interior $(200 \mathrm{~m})$ en las etapas de regeneración y adulta en los fragmentos de BST PNR El Vínculo y RNSC El Hatico, Valle del Cauca, Colombia.
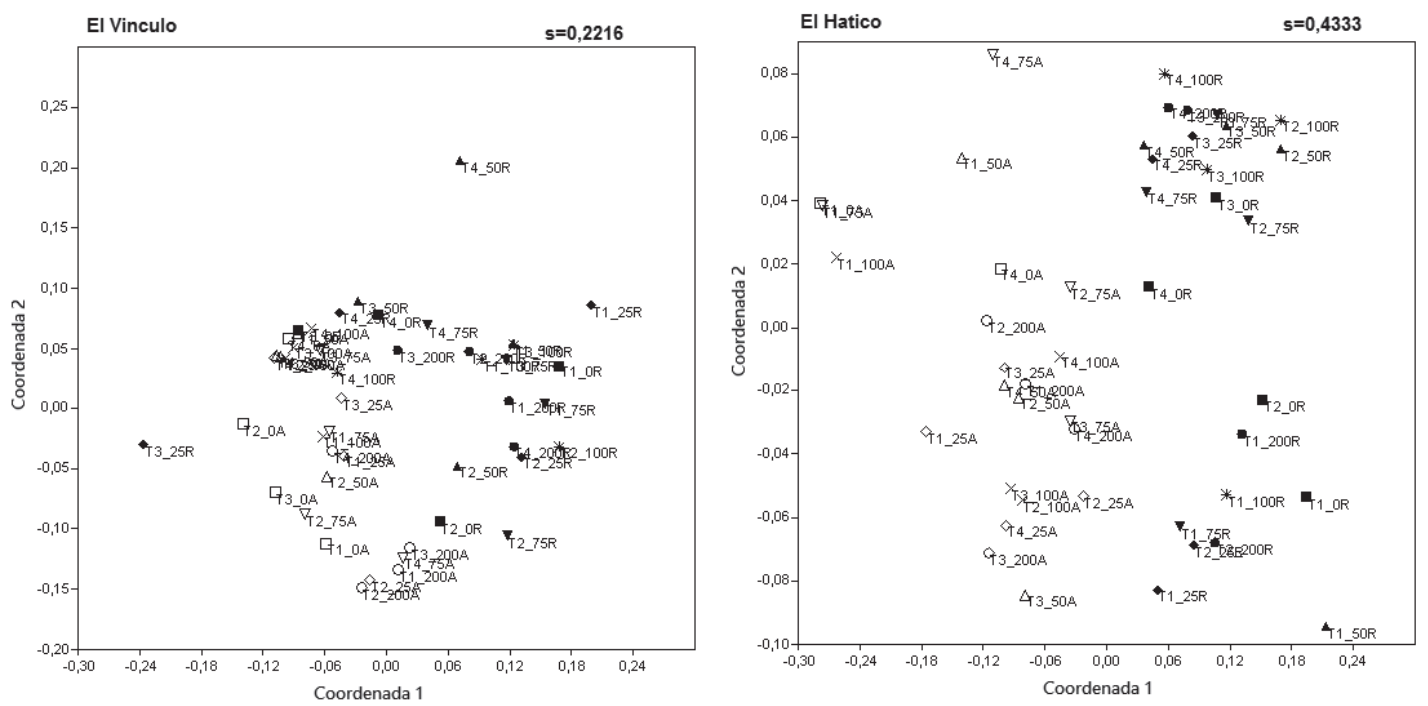

Figura 8. Comparación entre la composición de la etapa adulta y la etapa de regeneración, entre las parcelas de borde a interior en los fragmentos de BST del PRN El Vínculo y la RNSC El Hatico, Valle del Cauca, Colombia. Los símbolos sin relleno corresponden a la etapa adulta (A) y los símbolos llenos a la etapa de regeneración (R). $\square: 0$ m-A; $\mathbf{m}: 0 \mathrm{~m}-\mathrm{R}$; $25 \mathrm{~m}-\mathrm{A} ; \diamond: 25 \mathrm{~m}-\mathrm{R} ; \Delta: 50 \mathrm{~m}-\mathrm{A} ; \boldsymbol{\Delta}: 50 \mathrm{~m}-\mathrm{R} ; \nabla: 75 \mathrm{~m}-\mathrm{A} ; \boldsymbol{\nabla}: 75 \mathrm{~m}-\mathrm{R} ; \mathrm{x}: 100 \mathrm{~m}-\mathrm{A} ;{ }^{*}: 100 \mathrm{~m}-\mathrm{R} ; \circ: 200$ $\mathrm{m}-\mathrm{A} ; \bullet: 200 \mathrm{~m}-\mathrm{R}$. 


\section{Discusión}

Entre los impactos inmediatos y directos que ocurren con la formación de un fragmento de bosque, el más notable es la diferencia entre la estructura de la vegetación del borde y el interior (Harper et al., 2005). Esta diferencia obedece al daño y muerte de árboles por perturbaciones directas (i.e. viento, fuego, huracanes) o por la exposición repentina a las condiciones de una nueva matriz (i.e. agrícola, urbana, ganadera) (Saunders et al., 1991; Harper et al., 2005). Después de los impactos directos, se producen cambios en la estructura y composición de la vegetación que modifican la permeabilidad de los bordes respecto a las condiciones externas y los conducen a través de etapas de desarrollo temporal (Laurance et al., 2002). Dichas etapas comienzan con un aislamiento inicial debido a las pronunciadas diferencias con la matriz, luego un cierre del borde como producto del crecimiento de vegetación secundaria, y una estabilización final, independiente de las condiciones externas, que generalmente ocurre después de los cinco años en bosques tropicales (Williams-Linera, 1990; Laurance et al., 2002; Harper et al., 2005).

Teniendo en cuenta que el fragmento de bosque El Hatico fue preservado por sus dueños desde hace 70 años, sus bordes tienen el mismo tiempo de creación. Es factible que hayan pasado por las etapas de desarrollo de los bordes mencionadas por Laurance et al. (2002), y se encuentren en una etapa de estabilización ante las condiciones de la matriz, por lo que ya no ocurre la muerte y derribamiento de individuos adultos en el borde. Por otra parte, se considera que el efecto de la distancia sobre la altura y DAP de las plantas adultas en El Hatico, pueden ser debidas a las condiciones particulares de las parcelas y no a los impactos propios de la fragmentación reciente de un hábitat. Entre las particularidades de las parcelas de El Hatico, puede resaltarse la presencia de una población de G. angustifolia en uno de los transectos (transecto 1 , parcelas 0 a $100 \mathrm{~m}$ ), que está relacionada con los mayores valores de altura y DAP de la vegetación en estas parcelas. Estructuralmente, la población de guadua es dominante, su altura alcanza $24 \mathrm{~m}$, y posee una alta densidad de individuos que se diferencia de otras poblaciones de árboles dentro del bosque.

$\mathrm{Al}$ contrario de El Hatico, la estructura de la clase adulta en el fragmento El Vínculo no se diferenció entre las parcelas de borde a interior. Este bosque fue declarado como reserva hace 48 años y, en su totalidad, inició a partir de tierras dedicadas a la ganadería y la agricultura, que luego de un proceso de regeneración natural alcanzó un estado sucesional temprano (Torres et al., 2012). En consecuencia, la altura de la vegetación es muy homogénea a lo largo de todo el gradiente evaluado. Por otro lado, desde su declaración como reserva natural, el bosque ha estado rodeado por una matriz de ganadería y, de manera similar a El Hatico, los individuos en los bordes se han estabilizado ante las condiciones de la matriz que los rodea. Según las etapas de desarrollo temporal (Laurance et al., 2002), el proceso de regeneración que ocurre en los bordes disminuye el contraste entre el parche de bosque y la matriz, actuando como una banda protectora frente a los efectos de borde (Williams-Linera, 1990; Harper et al., 2005; Laurance et al., 2007).

Por otra parte, los individuos propios de la estructura original de un bosque pueden impulsar su proceso de recuperación, a pesar de haber sido degradado o apenas comenzar su proceso de sucesión. En procesos de rehabilitación, la inclusión de estos individuos vegetales ha sido implementada para potenciar la recuperación, ya que propician la llegada de dispersores de semillas y mejoran las condiciones para la emergencia de nuevas plantas (Albornoz, 2013; Corbin y Holl, 2012). Según nuestras observaciones, los árboles emergentes presentes en los fragmentos de bosque estudiados, están cumpliendo una función muy importante en la estructuración del dosel, la creación de un microclima para otras especies y el aporte de semillas y biomasa. Tanto en El Hatico como en El Vínculo, hubo mayor abundancia de juveniles en parcelas de interior, donde se 
encontraban aquellos árboles sobresalientes por sus mayores valores de altura y DAP. En El Hatico se registraron varios individuos de $A$. excelsum, $F$. insipida, C. pentandra, S. saman y S. sancona; y en El Vínculo, se registraron individuos de B. alicastrum y A. exelsum. Todas estas especies hacen parte de la composición típica del BST en el Valle de Cauca (Torres et al., 2012; Vargas 2012) y, además, las especies $B$. alicastrum, $S$. sancona y A. exelsum son consideradas emergentes de bosque seco primario (Silverstone-Sopkin, com. pers.).

Luego de las respuestas estructurales primarias que se dan con la creación de un borde, se presentan procesos de respuesta secundarios que involucran los procesos de regeneración, crecimiento, reproducción y mortalidad (Harper et al., 2005). Algunos estudios han encontrado que la densidad de plántulas disminuye con la cercanía al borde como resultado de la desecación cerca de los bordes o por la proliferación de especies de rápido crecimiento (Benítez-Malvido, 1998; Benítez-Malvido y Lemus-Albor, 2005). Por el contrario, los resultados de la presente investigación $\mathrm{y}$ de otros estudios realizados en BST muestran que el efecto de borde sobre la diversidad y estructura de la vegetación ha sido nulo o poco marcado (Sampaio y Scariot, 2011; Bouroncle y Finegan, 2011). Esta respuesta ha sido atribuida a las adaptaciones de las especies de BST a condiciones agrestes, causadas por la estacionalidad de lluvias de este ecosistema, y la presencia de dichas adaptaciones se ha resaltado como valor de conservación para estos fragmentos de bosque (Philips et al., 2006; Bouroncle y Finegan, 2011).

Los valores del $\mathrm{P}_{\text {Reg }}$ muestran que la población de plántulas y juveniles reflejan una baja proporción de los adultos presentes en El Vínculo (10-31 \%) y en El Hatico (8-15 \%). Esta medida es una proporción local que sería interesante comparar con el $\mathrm{P}_{\mathrm{Reg}}$ de otros BST, a partir de la medida del potencial de regeneración que se propone por primera vez en este estudio. En el caso de El Vínculo se cuenta con un estudio previo que permite comparar el estado actual de regeneración de este fragmento con su estado inicial (Rojas, 1991). En dicho estudio se realizó un monitoreo bianual entre 1972 y 1980, y sus resultados muestran que las cinco especies más abundantes en la etapa de regeneración fueron O. veraguensis, E. procera, A. pinnata, $M$. capote y Cytharexylum kunthianum (Rojas, 1991). Al comparar los resultados de ese monitoreo con la etapa de regeneración evaluada en la presente investigación, se observa que especies como $O$. veraguensis ha incrementado su abundancia; $\mathrm{y}$ otras como E. procera, ha disminuido en esta etapa, pero se destaca como la especie más abundante en estado adulto. En contraste, especies pioneras o heliófitas como C. kunthianum fueron abundantes en los inicios del Parque (Rojas, 1991), pero actualmente su presencia es muy baja y se restringe a los bordes. El aumento del reclutamiento y de la abundancia de adultos indica que el bosque ha ganado cobertura vegetal que favorece a especies de mayor tolerancia a la sombra, al mismo tiempo que muestra una dinámica positiva en la sucesión natural en esta área protegida (Torres et al., 2012).

Aunque los $\mathrm{P}_{\mathrm{Reg}}$ indican una baja representatividad de los adultos, al considerar el estado del que partieron estos bosques y que su conectividad con otros fragmentos está limitada, son de destacar los procesos de regeneración que se están dando en estos parches de bosque. Además, la presencia de juveniles demuestra que no solo germinaron los propágulos de la lluvia de semillas en forma de plántulas, sino que estas sobrevivieron a los filtros propios de cada especie (Grubb, 1977), y a la variabilidad ambiental dada por la estacionalidad del BST (Murphy y Lugo, 1986). Al evaluar las variaciones del potencial de regeneración respecto a la distancia al borde, solamente en El Vínculo, el potencial de regeneración fue menor en las parcelas de borde. Para ambos fragmentos, en el análisis de composición no se observaron agrupaciones de parcelas definidas por la distancia hacia el borde, y solo fue evidente la separación entre los grupos de la clase adulta y los de regeneración. Por una parte, la ausencia del efecto de borde sobre la etapa de regeneración (al igual que en la etapa adulta), puede ser debida al estado sucesional homogéneo de los bosques y a que el tiempo transcurrido desde la formación de 
los bordes no representa un cambio abrupto en las condiciones de establecimiento para las plántulas y juveniles. Por otro lado, los valores de potencial de regeneración y diferencias en la composición se calcularon en la misma unidad de muestreo para la etapa adulta y la de regeneración (i.e. $20 \mathrm{~m}^{2}$ ). Es posible que el área usada subestime la riqueza de especies de la clase adulta porque no incluye todas las especies presentes en el bosque. Dado lo anterior, para futuros estudios se sugiere usar parcelas de mayor tamaño para una evaluación más representativa de la etapa adulta, y dentro de estas delimitar subparcelas para la evaluación de las etapas de regeneración (Norden, 2007).

De acuerdo con nuestras observaciones, el proceso de regeneración natural está ocurriendo en los fragmentos evaluados, lo cual es de mucha importancia para su permanencia en el tiempo. Sin embargo, también debe tenerse en cuenta la necesidad que tienen estos fragmentos de expandirse hacia la matriz que los rodea. Si esto no ocurre, en el futuro la descendencia de varias especies quedará atrapada en pequeños fragmentos como si fueran "muertos vivientes" (Silverstone-Sopkin, 2011). En estos casos, la distribución de las especies dentro de los fragmentos está restringida por su limitada capacidad de dispersión, la carencia de sitios seguros para el establecimiento de plántulas o la ocurrencia de procesos denso-dependientes, en especial con la presencia de conespecíficos de la etapa adulta (Dewalt et al., 2015). Tal es el caso de especies como Laetia americana, Xylopia ligustrifolia y varias especies de la familia Amaryllidaceae, presentes en otros fragmentos de bosque seco del Valle del Cauca y ausentes en los fragmentos aquí estudiados (Lozano et al., 2007; SilverstoneSopkin, 2011).

Finalmente, cabe resaltar que uno de los mayores obstáculos para los procesos de regeneración en áreas por fuera de los fragmentos son las condiciones de la matriz que los rodea (Holl, 1999; Holl et al., 2000). En este estudio se presentó una marcada diferencia entre los parámetros de diversidad, estructura y potencial de regeneración entre las parcelas de la matriz y las parcelas de bosque. Se ha reportado que los principales limitantes para la regeneración en la matriz son la carencia de propágulos provenientes del bosque y la competencia con las gramíneas, además de otros factores como herbivoría, altas temperaturas, falta de nutrientes en el suelo y grado de compactación (Holl, 1999; Holl et al., 2000). Estos limitantes se manifiestan desde la etapa de dispersión, debido a la pérdida de viabilidad de las semillas o la depredación por ser más visibles en la matriz (Cubiña y Aide, 2001). Por este motivo, al incluir terrenos para la regeneración del bosque seco deberían de implementarse estrategias que favorezcan la dispersión de semillas hacia el exterior y mejoren las condiciones para la germinación y el establecimiento de plántulas (Holl et al., 2000; Cubiña y Aide, 2001).

\section{Conclusiones}

No se encontró un efecto de borde sobre la estructura, diversidad y potencial de regeneración de plantas leñosas en los dos fragmentos estudiados. Esta ausencia de efecto podría explicarse por el tiempo transcurrido desde la formación del borde (más de 40 años en los dos fragmentos), durante el cual los procesos de regeneración han homogenizado la vegetación entre los bordes y el interior de los fragmentos, y han favorecido una estabilización de la vegetación ante las condiciones de la matriz.

La medida del potencial de regeneración propuesta por primera vez en este estudio, representa una herramienta para estimar la representatividad de la etapa regeneración con relación a la etapa adulta. Esta medida también podría usarse para comparar el potencial de regeneración entre distintos bosques o para evaluar la variación de este potencial en el tiempo, bien sea ante la aparición de nuevas perturbaciones o, por el contrario, por la implementación de programas de conservación y restauración.

Los resultados de este estudio permiten documentar un proceso activo de regeneración 
natural en los fragmentos evaluados. Se ratifica la importancia de estos fragmentos como reservas de biodiversidad, que aportan a la permanencia del BST en el tiempo y representan una herramienta de investigación de las dinámicas que se dan en un ecosistema tan amenazado. Sin embargo, las matrices alrededor de estos fragmentos representan una barrera para su expansión y conectividad con otros fragmentos, por lo cual se hacen necesarias estrategias que favorezcan la dispersión de semillas hacia el exterior y optimicen las condiciones para la germinación y el establecimiento de los nuevos propágulos.

\section{Agradecimientos}

Los autores agradecemos a Camilo Giraldo y Daniel Jiménez por su apoyo en campo, así como a Édier Soto y Mauricio Posada por la identificación de los especímenes. También damos nuestros agradecimientos a los revisores anónimos por sus valiosas sugerencias para mejorar este artículo. Al Instituto para la Preservación del Patrimonio Cultural y Natural del Valle del Cauca (INCIVA) y a la familia Molina por su apoyo logístico durante la investigación y permiso de investigación. Este estudio fue financiado por Ecopetrol en su Tercera Convocatoria Nacional a la Biodiversidad "para la presentación de proyectos encaminados a la conservación de la diversidad biológica de los boques secos tropicales de Colombia y sus servicios ecosistémicos", como parte del proyecto Los bordes como amortiguadores de la degradación de los fragmentos de bosque seco tropical.

\section{Referencias}

Albornoz, F. E., Gaxiola, A., Seaman, B. J., Pugnaire, F.I. y Armesto J.J. (2013) Nucleationdriven regeneration promotes post-fire recovery in a Chilean temperate forest. Plant Ecology, 214, 765-776.

Arcila, A. M., Valderrama C. y Chacón, P. ( 2012). Estado de fragmentación del bosque seco de la cuenca alta del río Cauca, Colombia. Biota Colombiana, 13, 86-101.
Benítez-Malvido, J. (1998). Impact of forest fragmentation on seedling abundance in a tropical rain forest. Conservation Biology, 12(2), 380-389.

Benítez-Malvido, J. y Lemus-Albor, A. (2005). The seedling community of tropical rain forest edges and its interaction with herbivores and pathogens. Biotropica, 37, 301-313.

Botzat, A., Fischer, L., y Farwig, N. (2015). Regeneration potential in South African forest fragments: extinction debt paid off or hampered by contemporary matrix modification?. Plant Ecology, 216(4), 535-551.

Bouroncle, C. y Finegan, B. (2011). Tree regeneration and understory woody plants show diverse responses to forest-pasture edges in Costa Rica. Biotropica, 43(5), 562-571.

Brandão, J. D. F. C., Martins, S. V. y Brandão, I. J. (2016). Potencial de regeneração de uma área invadida por Pteridium aquilinum (L.) Kuhn no parque nacional do caparaó. Floresta, 46(4), 543552.

Cavender-Bares, J. y Bazzaz, F. (2000). Changes in drought response strategies with ontogeny in Quercus rubra: implications for scaling from seedlings to mature trees. Oecologia, 124, 8-18.

Chen, J., Saunders, S., Crow, T., Naiman, R., Brosofske, K., Mroz, G., Brookshire, B., Franklin, J. F. (1999) Microclimate in Forest Ecosystem and Landscape Ecology: Variations in local climate can be used to monitor and compare the effects of different management regimes. BioScience, 49 (4), 288-297, https://doi.org/10.2307/1313612

Comita, L. S., Queenborough, S. A., Murphy, S. J., Eck, J. L., Kaiyang, X., Krishnadas, M., Beckman, N. y Zh, Y. (2014). Testing predictions of the JanzenConnell hypothesis: a meta-analysis of experimental evidence for distance and densitydependent seed and seedling survival. Journal of Tropical Ecology, 102, 845-856.

Corbin, J. D. y Holl, K. D. (2012). Applied nucleation as a forest restoration strategy. Forest Ecology and Management, 265, 37-46. 
Cubiña, A. y Aide M, T. (2001). The effect of distance from forest edge on seed rain and soil seed bank in a tropical pasture. Biotropica, 33(2), 260-267.

Dewalt, S. J., Taylor, B. N. y Ickes, K. (2015). Density-dependent Survival in Seedlings Differs among Woody Life-forms in Tropical Wet Forests of a Caribbean Island. Biotropica, 47, 310-319.

Fahrig, L. (2003). Effects of habitat fragmentation on biodiversity. Annual review of ecology, evolution, and systematics, 34(1), 487-515.

Faraway, J. (2016). Extending the linear model with R: generalized linear, mixed effects and nonparametric regression models. USA: CRC Press. 312 pp.

Fischer, J. y Lindenmayer, D. B. (2007). Landscape modification and habitat fragmentation: a synthesis. Global ecology and biogeography, 16(3), 265-280.

Fletcher, Jr., Ries, R. J., Battin, L. y Chalfoun, A. D. (2007). The role of habitat area and edge in fragmented landscapes: definitely distinct or inevitably intertwined? Canadian journal of zoology, 85 (10), 1017-1030.

García, H., Corzo, G., Isaacs, P. y Etter, A. (2014). Distribución y estado actual de los remanentes del bioma de bosque seco tropical en Colombia: insumos para su gestión. Pp: 229-241. En Pizano, C. y García, H. El Bosque Seco Tropical en Colombia (349 pp). Bogotá, D.C., Colombia: Instituto de Investigación de Recursos Biológicos Alexander von Humboldt.

Grubb, P. J. (1977). The maintenance of speciesrichness in plant communities: the importance of the regeneration niche. Biological reviews, 52(1), 107-145.

Hammer, Ø., Harper, D. A. T. y Ryan, P. D. (2001). Paleontological Statistics Software: Package for Education and Data Analysis. Paleontologia Electronica.

Harper, K. A., Macdonald, S. E., Burton, P. J., Chen, J., Brosofske, K. D, Saunders, S. C., y Esseen,
P. A. (2005). Edge influence on forest structure and composition in fragmented landscapes. Conservation Biology, 19(3), 768-782.

Holl, K. D. (1999). Factors limiting tropical rain forest regeneration in abandoned pasture: seed rain, seed germination, microclimate, and soil. Biotropica, 31(2), 229-242.

Holl, K. D., Loik, M. E, Lin, E. H. YSamuels I. A. (2000). Tropical montane forest restoration in Costa Rica: overcoming barriers to dispersal and establishment. Restoration ecology, 8(4), 339349 .

Janzen, D. H. (1988). Management of Habitat Fragments in a Tropical Dry Forest: Growth. Annals of the Missouri Botanical Garden, 75(1), 105116.

Laurance, W. F., Lovejoy T. E., Vasconcelos H. L., Bruna E. M., Didham, R. K., Stouffer, P. C., y Sampaio E. ( 2002). Ecosystem decay of Amazonian forest fragments: a 22 - year investigation. Conservation Biology, 16(3), 605618.

Laurance, W. F., Nascimento, H. E., Laurance, S. G., Andrade, A. C., Fearnside, P. M., Ribeiro, J. E., y Capretz, R. L. (2006). Rain forest fragmentation and the proliferation of successional trees. Ecology, 87 (2), 469-482.

Lopes, A. V., Girão, L. C., Santos, B. A., Peres, C. A. y Tabarelli, M. (2009). Long-term erosion of tree reproductive trait diversity in edge-dominated Atlantic forest fragments. Biological Conservation, 142 (6), 1154-1165.

Lozano, F. H., Vargas, W., Caicedo, P. C., Jiménez, E., Guerra, G. y Cardona, C. A. (2007). Herramientas de manejo del paisaje para favorecer el mantenimiento y la restauración del bosque seco tropical en el valle geográfico del río cauca. Informe para el Instituto de Investigación de Recursos Biológicos Alexander von Humboldt.

Miles, L., Newton, A. C., DeFries, R. S., Ravilious, C., May, I., Blyth, S., y Gordon, J. E. (2006). A global overview of the conservation status of 
tropical dry forests. Journal of Biogeography, 33(3), 491-505.

Mok, H.-F., Arndt, S. K. y Nitschke, C. R. (2012). Modelling the potential impact of climate variability and change on species regeneration potential in the temperate forests of South-Eastern Australia. Global Change Biology, 18, 1053-1072. doi:10.1111/j.13652486.2011.02591.x

Molina, C., Molina, C., Molina, E. y Molina, J. (2012). Manejo agroecológico de caña de azúcar y sistemas silvopastoriles intensivos Alternativas sostenibles para el valle geográfico del río Cauca. Reserva Natural El Hatico. RevistaTecnicaña, 29, 28-35.

Murcia, C. (1995). Edge effects in fragmented forests: implications for conservation. Trends in ecology \& evolution, 10(2), 58-62.

Murphy, P. G., y Lugo, A. E. (1986). Ecology of tropical dry forest. Annual Review of Ecology and Systematics, 17, 67-88.

Norden, N., Chave, J., Caubere, A., Chatelet, P., Ferroni, N., Forget, P. M. y Thébaud, C. (2007). Is temporal variation of seedling communities determined by environment or by seed arrival? A test in a neotropical forest. Journal of Ecology, 95(3), 507-516.

Norden, N. (2014). Del porqué la regeneración natural es tan importante para la coexistencia de especies en los bosques tropicales. Colombia Forestal, 17(2), 247-261.

Phillips, O. L., Rose, S., Mendoza, A. M., y Vargas, P. N. (2006). Resilience of southwestern Amazon forests to anthropogenic edge effects. Conservation Biology, 20(6), 1698-1710.

Pizano, C. y García, H. (2014). El bosque seco tropical en Colombia. Bogotá, D.C., Colombia: Instituto de Investigación de Recursos Biológicos Alexander von Humboldt.

Poorter, L. y Markesteijn, L. (2008). Seedling traits determine drought tolerance of tropical tree species. Biotropica, 40(3), 321-331.
Ries, L., Fletcher, Jr. R. J., Battin, J. y Sisk, T. D. (2004). Ecological responses to habitat edges: mechanisms, models, and variability explained. Annual Review of Ecology, Evolution, and Systematics, 35, 491-522.

R Core Team. (2014). R: a language and environment for statistical computing, $\mathrm{R}$ Foundation for Statistical Computing, Vienna, Austria, available at: www.r-project.org (accesed September 2015).

Rojas, O. (1991). Evolución de una sucesión vegetal en el Valle del Cauca: Generalidades sobre el estudio de la dinámica de regeneración en el Santuario de El Vínculo. Cespedesia, 13 (49-50), 152-221.

Sampaio, A. B. y Scariot, A. (2011). Edge effect on tree diversity, composition and structure in a deciduous dry forest in central Brazil. Revista Árvore, 35 (5), 1121-1134.

Saunders, D. A., Hobbs, R. J. y Margules, C. R. (1991). Biological consequences of ecosystem fragmentation: a review. Conservation Biology, 5 (1), 18-32.

Silverstone-Sopkin, P. A. (2011). Los muertos vivientes: la historia natural de cuatro lirios amazónicos del suroccidente de Colombia. 100 pp. Cali, Colombia: Programa Editorial, Universidad del Valle.

Sizer, N., y Tanner, E. V. (1999). Responses of woody plant seedlings to edge formation in a lowland tropical rainforest, Amazonia. Biological Conservation, 91(2), 135-142.

Torres, A. M., Adarve, J. B., Cárdenas, M., Vargas, J .A., Londoño, V., Rivera, K., Home, J., Lucía, O. y María, Á. (2012). Dinámica sucesional de un fragmento de bosque seco tropical. Biota Colombiana, 13, 66-85.

Vargas-Rodriguez, Y. L., Vázquez-García, J. A. y Williamson, G. B. (2005). Environmental correlates of tree and seedling-sapling distributions in a Mexican tropical dry forest. Plant Ecology, 180, 117-134. 
Vieira, D. L. y Scariot, A. (2006). Principles of natural regeneration of Tropical Dry Forests for regeneration. Restoration Ecology, 14, 11-20.

Williams-Linera, G. (1990). Vegetation Structure and Environmental Conditions of Forest Edges in Panama. The Journal of Ecology, 78, 356-373.
Williams-Linera, G. y Lorea, F. (2009). Tree species diversity driven by environmental and anthropogenic factors in tropical dry forest fragments of central Veracruz, México. Biodiversity and Conservation, 3269-3293.

Anexo 1. Especies registradas en los dos fragmentos de bosque seco tropical (PNR El Vínculo y El Hatico) en el estado de adulto (A) y en las dos etapas de sucesión, juvenil (J) y plántula (P), y lugar de regeneración (LR) de especies indicadoras en la matriz (M), borde (B), interior (I). Disponible en línea: http://revistas.humboldt.org.co/index.php/biota/rt/suppFiles/552/0

\section{Natalia Henao}

Universidad del Valle

Cali, Colombia

henao.natalia@correounivalle.edu.co

\author{
Alba Marina Torres \\ Universidad del Valle \\ Cali, Colombia \\ alba.torres@correounivalle.edu.co
}

Juan Carlos Tafur

Universidad del Valle

Cali, Colombia

jcarlostafur@gmail.com

\section{Leonardo Guevara}

Universidad del Valle

Cali, Colombia

leonardo.guevara@correounivalle.edu.co
$¿$ Existe un efecto de borde sobre la estructura vegetal y el potencial de regeneración en fragmentos de bosque seco tropical?

Citación del artículo: Henao, N., Torres, A. M., Tafur, J. C. y Guevara, L. (2018). ¿Existe un efecto de borde sobre la estructura vegetal y el potencial de regeneración en fragmentos de bosque seco tropical? Biota Colombiana, 19(1), 3-20. DOI: 10.21068/c2018.v19n01a01.

Recibido: 25 de noviembre de 2015

Aprobado: 1 de mayo de 2018 